\title{
THE STREPTOCOCCAL ANTIFIBRINOLYSIN TEST IN CLINICAL USE ${ }^{1}$
}

\author{
BY PAUL L. BOISVERT \\ (From the Department of Pediatrics, Yale University School of Medicine and the Pediatric \\ Service of the New Haven Hospital and Dispensary, New Haven)
}

(Received for publication August 16, 1939)

The streptococcal antifibrinolysin test of Tillett and Garner (1) had promise of being a practical serological method for the diagnosis of a recent hemolytic streptococcal infection. The test is simple to perform, inexpensive, and uniform in its results. In the experience of Tillett and his associates $(2,3)$, the test was generally positive in diseases caused by the hemolytic streptococcus, and negative in non-streptococcal infections. Subsequent observations by other investigators, however, indicated that the antifibrinolysin test is non-specific. Myers, Keefer and Holmes (4) obtained strongly positive tests in 2 of 6 patients with gonococcal arthritis, and in 2 cases of bacterial endocarditis due to the streptococcus viridans. Waaler $(5,6)$ found the test to be positive in 3 of 4 patients with streptococcus viridans bacteremia, and in 6 patients with non-streptococcal pneumonia. He concluded that the presence of a positive test is of little diagnostic value.

The antifibrinolysin test has been used as a laboratory aid in our pediatric clinic for over 3 years. Our early experience with the test corroborated the findings of Tillett and associates. Although in very young infants variable results were obtained, we found the test generally to be positive in children recovering from a hemolytic streptococcal infection, and negative in other types of infectious disease. Furcolow and Fousek (7) of this clinic reported in 1936 the results of 84 tests on 70 patients. The positive tests numbered 37 , and 36 of these were from definite cases of streptococcal infection; the exception was a patient with staphylococcal bacteremia. Of the negative tests none was from cases of hemolytic streptococcal disease. As our series increased, we occasionally encountered positive tests in nonstreptococcal infections without an associated bacteremia but we were inclined to view the positive results in these cases as an indication of a recent mild streptococcal infection. In many of the

\footnotetext{
1 Presented in part at the 1938 meeting of The Society for Pediatric Research.
}

children there was a history of a recent infection which could have been caused by the hemolytic streptococcus. We did not feel that the rather frequent association of positive tests with a bacteremia interfered with the practical value of the method so long as we were aware that such an exception existed. Such cases were not common and the presence of organisms in the blood stream was easily demonstrated by culture. It did not seem fair to regard the test as non-specific on this basis since in the presence of a bacteremia it might be expected that all of the body's methods of defense might be brought into play.

The specificity of the test in pediatric patients first became open to our suspicion during the pneumococcal pneumonia season of 1936 to 1937 . Almost all these patients with pneumonia showed strongly positive antifibrinolysin tests at the time of admission to the hospital. In the large majority of these cases, there was no history of recent illness, and no clinical or bacteriological evidence of a coincidental hemolytic streptococcal infection. None of the patients received serum. This seemingly definite association of positive antifibrinolysin tests with a specific type of nonstreptococcal disease induced us to study the behavior of the test throughout the course of this disease and in those due to the hemolytic streptococcus. Later, all available types of infectious disease were studied in this respect since it became apparent, as the test came into routine use, that its non-specificity was not limited to pneumococcal pneumonia.

This report represents a study of the trend of repeated antifibrinolysin tests in 15 normal children, 6 newborns, and 203 patients of the pediatric age-group. ${ }^{2}$

\section{METHODS OF STUDY}

During a period of over a year repeated antifibrinolysin tests were performed on all patients

\footnotetext{
2 Children up to 16 years of age are admitted to the pediatric service.
} 
on the pediatric service of New Haven Hospital with definite hemolytic streptococcal infections and with various types of non-streptococcal disease. The non-streptococcal disease group of patients was made up of those in whom there was no evidence, clinical or bacteriological, of recent contact with the hemolytic streptococcus. Included in the study were 15 normal infants and children, and 6 newborns.

Blood for testing was taken at the time of the patient's admission and at 2- to 4-day intervals throughout the period of hospitalization. In a few of the patients, where convalescence did not seem complete at the time of discharge, samples of blood were taken upon their return to the out-patient clinic for follow-up examinations. Each of the 15 normal children was tested at 3- and 4-day intervals during the first week of observation, and then at weekly intervals for a month. Studies on this group covered all seasons of the year. Samples of blood were obtained from the 6 newborns during the first twentyfour hours of life, at the end of 1 week, and on the tenth day of life (time of discharge). Tests were repeated on 3 of the 6 at the time of the routine 6 weeks' clinic check-up.

Antifibrinolysin test. The method is that of Tillett and Garner (1) and is based upon their observation that human hemolytic streptococci produce a substance (fibrinolysin) which will dissolve the plasma-clot of normal individuals, and that the blood of patients recovering from a hemolytic streptococcal infection generally shows resistance (antifibrinolysin) to this lytic action of the organism.

Collection of blood. Three cc. of blood to be tested was obtained by venipuncture and placed in a sterile $11.0 \times 100.0 \mathrm{~mm}$. glass tube containing 6.0 milligrams of potassium oxalate $(0.3 \mathrm{cc}$. of a 2 per cent solution of oxalate in distilled water). The tube was corked and inverted several times to prevent clotting. The tube was then placed in the ice box until used for the test, which was always done within 48 hours after the blood was taken.

Source of fibrinolysin. Ro, a strain of hemolytic streptococcus isolated in this laboratory from the blood of a child with a fatal septicemia, was used for all the tests. The culture was maintained by daily transfers in beef heart infusion broth containing 0.05 per cent dextrose. This strain has been used daily for about 2 years and regularly lyses the plasma-clot of a normal individual in 15 minutes or less. The fresh culture was routinely tested at about weekly intervals for maintenance of fibrinolytic activity. No variation great enough to affect the test was observed. In our earlier work, duplicate tests were run using a strain of hemolytic streptococcus from a case of scarlet fever as a control. The results were so uniform, however, that for simplicity only strain Ro was used in this study. From time to time we have also employed the patient's own strain of hemo- lytic streptococcus as a source of fibrinolysin for the test. Again, there was no significant difference between the results with Ro and with the homologous strain.

Description of test. The oxalated sample of blood was centrifuged for 3 to 4 minutes at about 1500 r.p.m., and $0.2 \mathrm{cc}$. of plasma was removed and diluted with $0.8 \mathrm{cc}$. of sterile physiological salt solution. To this was added $0.5 \mathrm{cc}$. of an 18-hour broth culture of the hemolytic streptococcus. Lastly, $0.25 \mathrm{cc}$. of calcium chloride ( 0.25 per cent solution in normal salt solution) was added, and the thoroughly mixed contents of the tube were immediately incubated in a water bath at $37.5^{\circ} \mathrm{C}$. In about 10 minutes a solid opaque clot was formed. The interval between the time of clotting and that of complete liquefaction was taken to indicate the degree of resistance of the patient's plasma to the fibrinolytic action of the hemolytic streptococcus. This resistance (amount of antifibrinolysin) was graded as follows:

$4+$ indicates no lysis of the plasma-clot in 24 hours. $3+$ indicates complete lysis in 8 to 24 hours.

$2+$ indicates complete lysis in 3 to 8 hours.

$1+$ indicates complete lysis in 1 to 3 hours.

- indicates complete lysis in less than 1 hour.

In the text, a positive test refers to a $1+$ to $4+$ reaction. Tests were terminated at 24 hours since clots which remained solid for this length of time continued so for days.

Bacteriological studies. Fresh beef heart infusion medium is used routinely in the pediatric bacteriological laboratory. Cultures of the nose, throat, blood, and material from purulent lesions (spinal fluid, pleural fluid, aural discharge, mastoid pus, etc.) were taken at the time of the patient's admission and subsequently as seemed indicated. Colonies from any of the above sources which resembled those of the hemolytic streptococcus were tested for bile insolubility and production of a soluble hemolysin for rabbit's erythrocytes, and were then grouped by the serological method of Lancefield $(8,9$, 10).

\section{RESULTS}

Early in the study it became apparent that the trend of repeated antifibrinolysin tests in pneumococcal pneumonia was different from that generally seen in hemolytic streptococcal infections. In streptococcal diseases the tests were generally negative during the acute stage of the illness and became increasingly positive at about the time of recovery. In pneumococcal pneumonia the strongly positive tests, which were commonly encountered, were present only during the acute febrile stage of the infection and subsequent tests showed a rapid decrease to negative. To demonstrate this difference in the trend of the tests, charts of temperature course and antifibrinolysin 
test results accompanied by a brief clinical summary are given for a typical case of pneumococcal pneumonia (Chart 1) and of a hemolytic streptococcal infection, scarlet fever (Chart 2). Both patients were of the same age and were hospitalized on the same day of illness.

As the study was enlarged to include many of the common types of non-streptococcal infection and a larger group of hemolytic streptococcal discases, a total of 6 antifibrinolysin test trends was observed:

$A$. Negative tests which persisted throughout the patient's illness and convalescence (Chart $3)$.

$B$. Positive tests during the acute febrile stage of an infection which rapidly changed to negative ones (Chart 1 ).

$C$. Negative tests during the acute stage of the disease which became increasingly positive during the period of convalescence (Chart 2).

$D$. Strongly positive tests which persisted throughout the patient's illness and convalescence (Chart 4 ).
$E$. Positive tests during the acute illness which changed to negative and then reverted to positive (Chart 5).

$F$. Negative tests which rapidly changed to positive and then back to negative (Chart 6 ).

For simplicity these 6 trends are represented graphically in Chart 7 and will subsequently be referred to by letter and a descriptive phrase, e.g. Trend A (persistently negative). When the results are plotted it becomes more than ever apparent that there is a definite trend in the course of the tests and that the trend is a smooth one without gross fluctuations.

The 6 antifibrinolysin trends and the conditions in which they were found are summarized in Chart 8.

The group of " normal" individuals consisted of 5 infants and 10 children who were apparently in good health and free from any type of infection. These subjects varied from 3 months to 16 years of age. The 6 healthy newborns who were included in the study are tabulated separately since their antifibrinolysin trend was found to be distinctly different from that of the older infant.

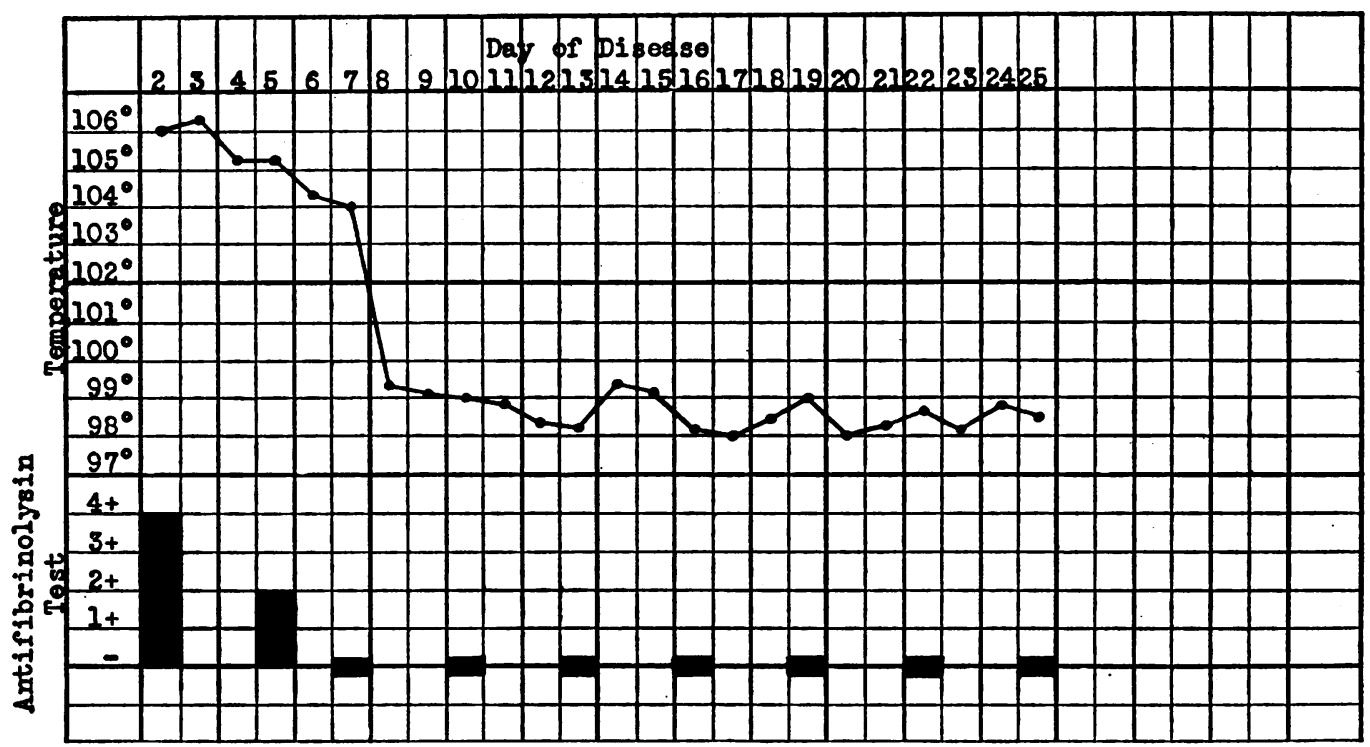

Chart 1. Patient G. J. Age 3 Years. Diagnosis: Pneumococcal Pneumonia, Type I

Child was admitted on the second day of the disease with physical and roentgenological signs of right lower lobe pneumonia. Pneumococcus type I was the predominating organism in the nose and throat cultures; there were no hemolytic streptococci. Child remained very ill until the seventh day when his temperature dropped to normal by crisis. His subsequent course was satisfactory.

Antifibrinolysin tests. Test was strongly positive on admission. It was still positive on the fifth day of the disease but to a lesser degree. With the fall in temperature on the seventh day, it became negative. Subsequent tests were also negative. 


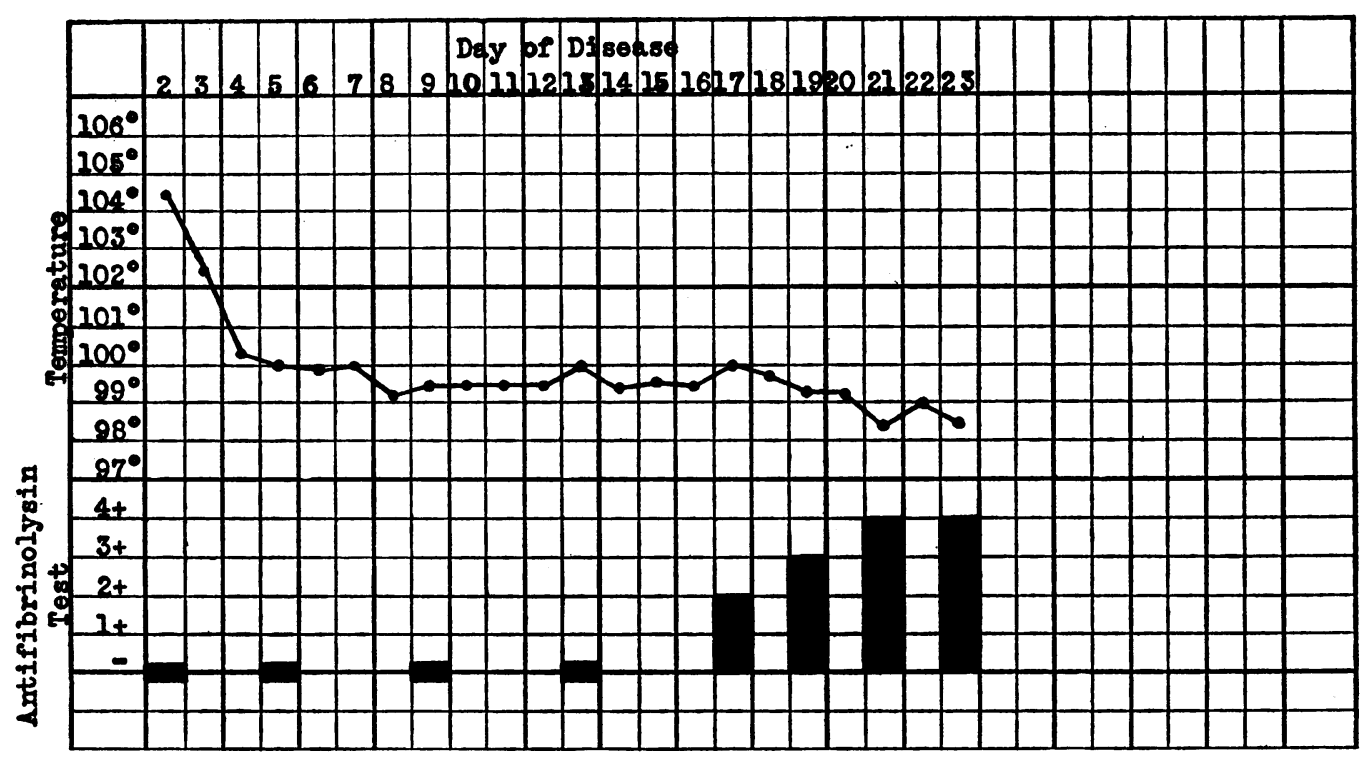

Chart 2. Patient M. D. Age 3 Years. Diagnosis: Scarlet Fever

Child was admitted on the second day of illness with classical signs of scarlet fever of moderate severity. Throat culture showed a predominance of hemolytic streptococci. Child's temperature dropped rapidly toward normal, recovery was uneventful and desquamation occurred.

Antifibrinolysin tests. Tests were negative throughout the active stage of the disease and became increasingly positive during convalescence.

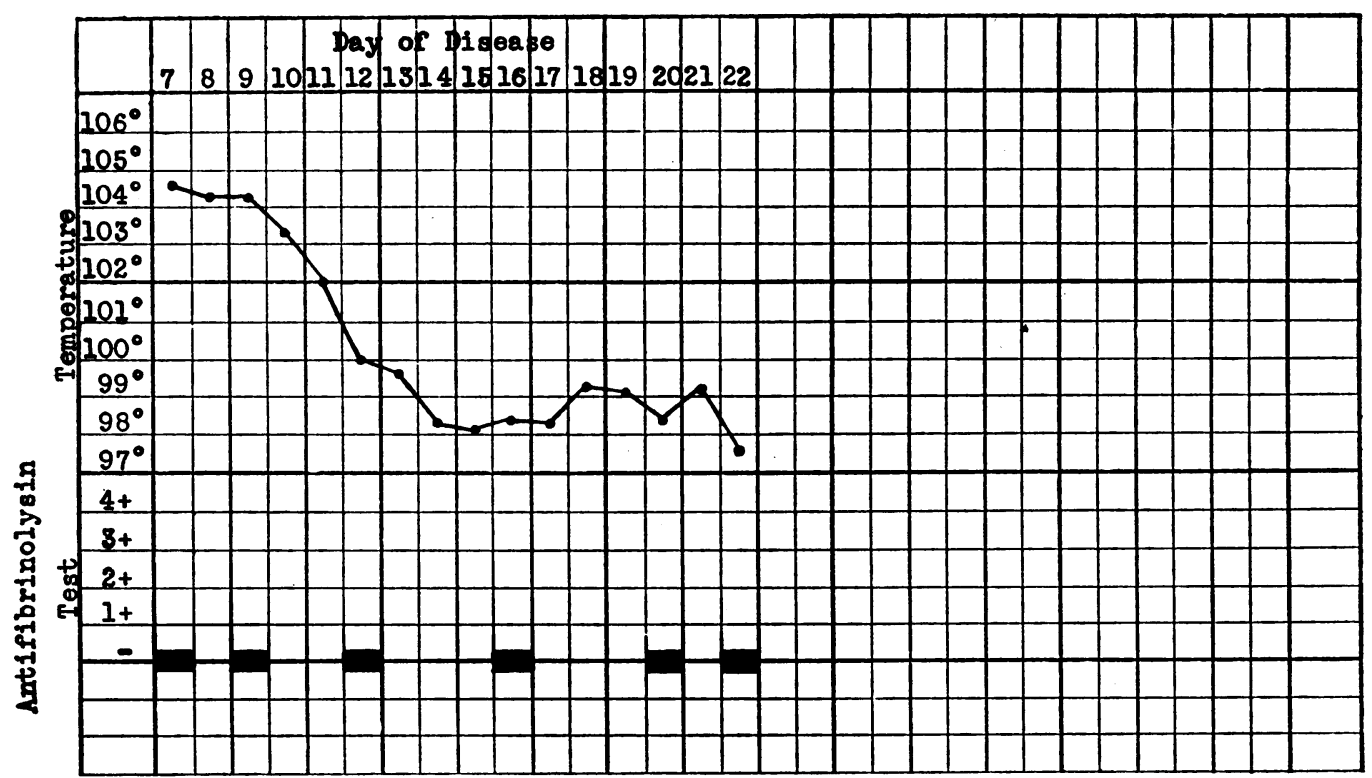

Chart 3. Patient M. M. Age 3 Years. Diagnosis: Pyelitis (B. Coli)

Child was admitted on the seventh day of the disease with a history of high fever, urinary frequency, and abdominal pain. Physical examination was negative except for costovertebral angle tenderness on the right side. Urine showed a 3 plus albumin and numerous white blood cells. B. coli was cultured from the urine. Nose and throat cultures were negative for hemolytic streptococci. Under sulfanilamide therapy child's temperature dropped to normal on the thirteenth day and remained within normal limits. The urine cleared coincidentally with the fall in temperature.

Antifibrinolysin tests. Tests were persistently negative throughout the child's illness and convalescence. 


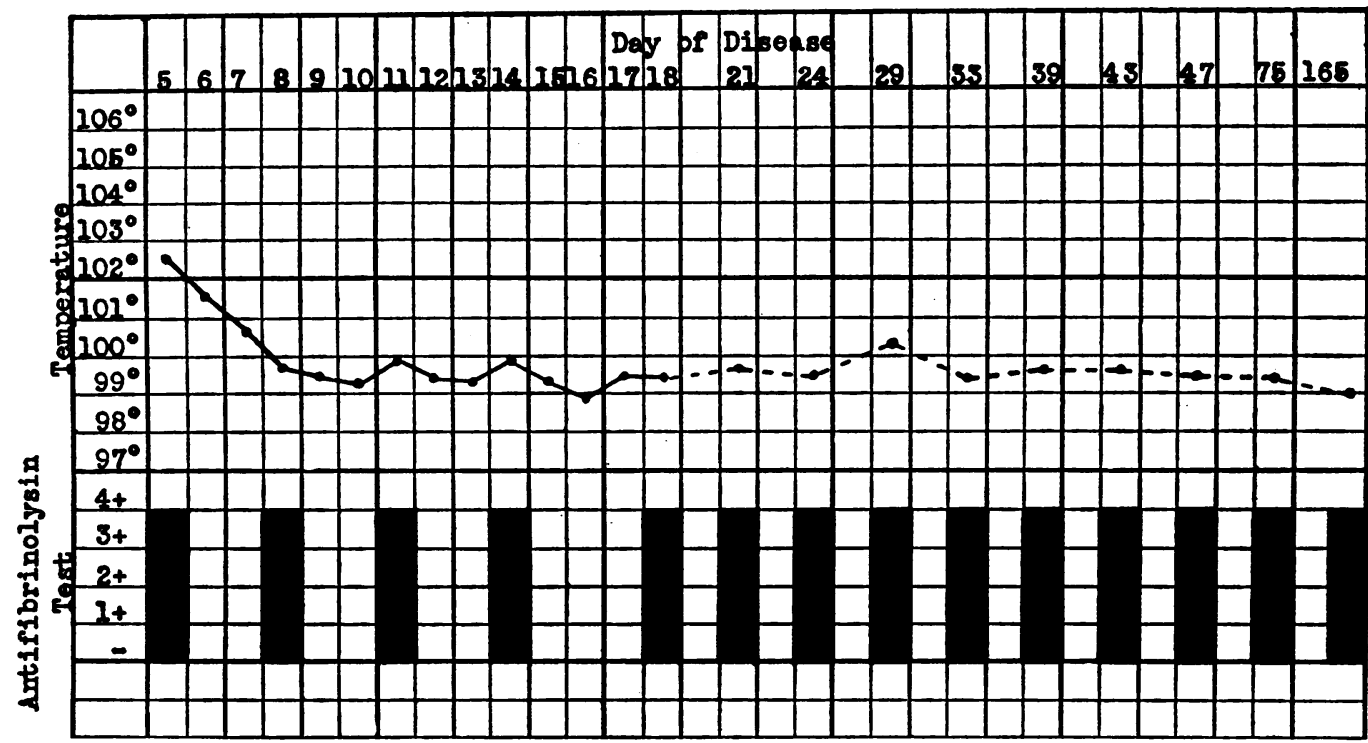

Chart 4. Patient A. S. Age 13 Years. Diagnosis: Rheumatic Fever, Rheumatic Heart Disease

Child was admitted on the fifth day of the disease with fever, epistaxes and pains in her knees. The heart was overactive and the knees were swollen and tender. The sedimentation rate was $26 \mathrm{~mm}$. per hour. Nose and throat cultures showed a predominance of hemolytic streptococci until the twentyseventh day of illness. Subsequent cultures were negative. Signs of rheumatic activity persisted until the thirty-ninth day. Child was transferred on the forty-seventh day to the Children's Community Center for convalescence. In the meantime, signs of a mitral and possibly an aortic heart lesion had appeared.

Antifibrinolysin tests. Tests were persistently positive during the entire period of hospitalization. Repeat tests 1 and 4 months after child's discharge were still strongly positive although her convalescence was uncomplicated.

There were 75 cases of hemolytic streptococcal infection. Seventy of these were primary infections and include scarlet fever ( 25 cases), erysipelas ( 7 cases), acute tonsillitis and cervical adenitis (25 cases), otitis media and mastoiditis (10 cases), pneumonia ( 1 case), meningitis (1 case), and cellulitis ( 1 case). In 3 cases the hemolytic streptococcal infection followed a nonstreptococcal disease: tuberculous pneumonia later complicated by hemolytic streptococcal tonsillitis and purulent otitis media which was followed by nephritis (1 case), pneumococcal pneumonia followed during the period of convalescence by hemolytic streptococcal tonsillitis and later acute hemorrhagic nephritis ( 1 case), and B. coli infection of the urinary tract complicated by hemolytic streptococcal tonsillitis and cervical adenitis (1 case). Both of the remaining 2 patients appeared from their course to be straightforward cases of scarlet fever. However, the presence of patho- genic pneumococci-type VII in one instance and type XIV in the other-in the nose and throat of these 2 patients suggests the possibility of a recent or simultaneous pneumococcal infection.

Thirty cases of acute rheumatic fever, 3 of chorea, and 11 of acute hemorrhagic nephritis were studied in view of the possible or probable relationship of the hemolytic streptococcus to these diseases.

In the non-streptococcal disease group, upper respiratory infections ( 6 cases), influenza ( 5 cases), pneumococcal pneumonia ( 32 cases), and poliomyelitis ( 7 cases) are tabulated separately as they were the most common types of non-streptococcal infection encountered during the study. There were 34 patients with other varieties of non-streptococcal disease. Of these, 20 are classified as " mild" and 14 as "severe," depending on the severity of the illness in the particular patient. The "mild" infections include pertussis 


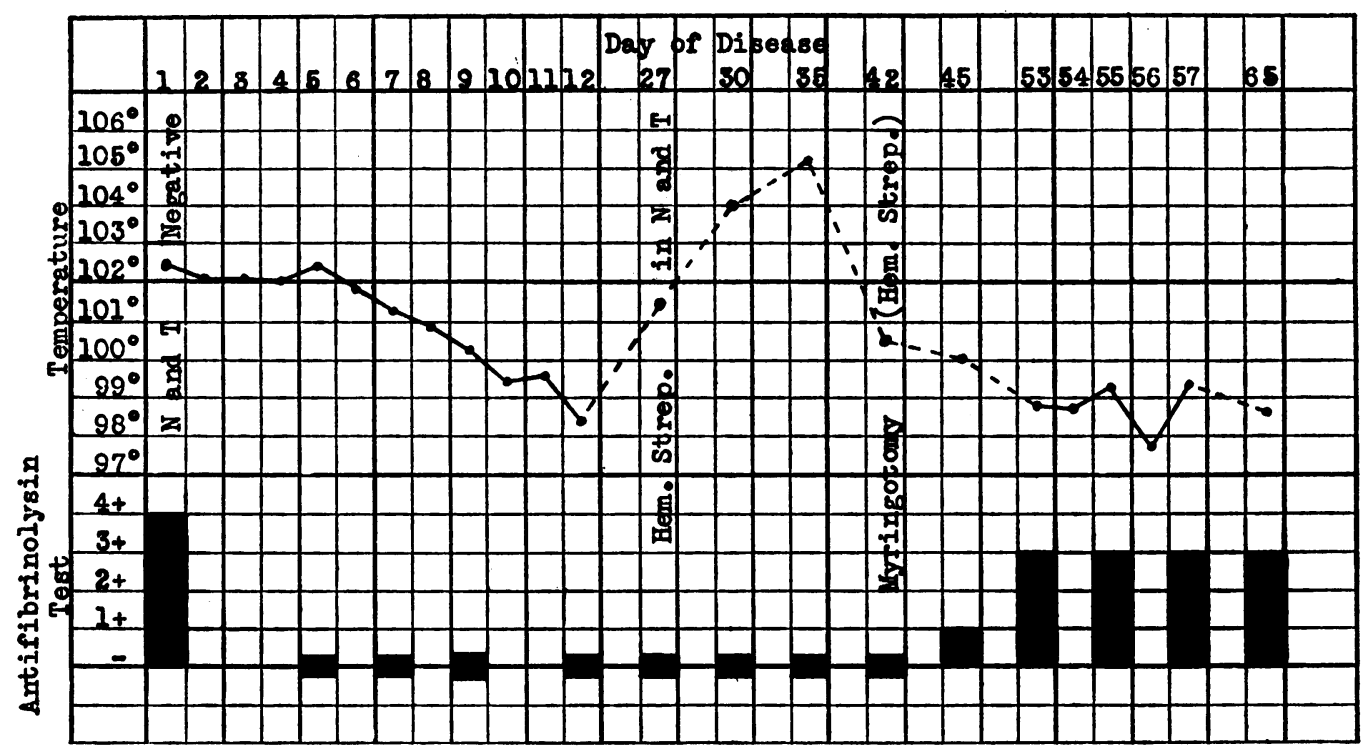

Chart 5. Patient T. U. Age 14 Months. Diagnosis: Tuberculous Pneumonia with Effusion; Acute Pharyngitis, Cervical Adenitis and Bilateral Purulent Otitis Media (Hemolytic Streptococcal); Acute Hemorrhagic Nephritis

Baby was admitted on apparently the first day of illness with physical and roentgenological signs of pulmonary tuberculosis with pleural effusion on the right side. Tuberculin test was strongly positive. Parents and 4 siblings had active tuberculosis. A thoracentesis yielded sterile straw-colored fluid. On the twenty-seventh day, the baby developed profuse nasal discharge, fiery red throat, and enlarged cervical glands. Nose and throat cultures, which were previously negative for pathogens, showed a predominance of hemolytic streptococci. On the forty-second day the right ear began to discharge and a left myringotomy was performed. Hemolytic streptococci were cultured from the pus from both ears. Urine at that time showed albumin and red blood cells. The streptococcal infection and the nephritis gradually subsided. The baby was discharged to a sanitorium because of persistence of the tuberculous process.

Antifibrinolysin tests. Test was strongly positive during the acute phase of the tuberculous pneumonia but rapidly became negative. Tests remained negative during the acute febrile stage of the hemolytic streptococcal infection but became strongly positive during convalescence.

( 3 cases), bronchitis ( 4 cases), chicken pox ( 1 case), mumps ( 1 case), bronchopneumonia (5 cases), urinary tract infection ( 2 cases), diphtheria ( 1 case), gonococcal vaginitis ( 1 case), measles ( 1 case), and exanthem subitum ( 1 case). The " severe" infections consist of staphylococcal septicemia (1 case), typhoid fever (3 cases), bacillary dysentery ( 2 cases), meningococcus meningitis ( 2 cases), pulmonary tuberculosis ( 1 case), pyelitis due to B. coli ( 2 cases), encephalitis (1 case), pneumococcal empyema (1 case), and streptococcus viridans septicemia (1 case).

Antifibrinolysin Trend A (persistently negative) was present in all of the 15 normal infants and children. It was also found in all of the cases of mild and in some of the cases of severe non-streptococcal infection. It occurred occasionally in a hemolytic streptococcal illness and in acute rheumatic fever, chorea and acute nephritis.

Trend $\mathrm{B}$ (positive to negative) was frequently observed in the more severe non-streptococcal diseases, notably pneumococcal pneumonia, in all the newborns, and occasionally in patients with a hemolytic streptococcal infection.

Trend C (negative to positive) occurred in $\mathbf{5 3}$ of the 70 cases of primary hemolytic streptococcal disease, and was seen in no other condition.

Trend D (persistently positive) was found in most of the cases of rheumatic fever, chorea and acute nephritis, in a few cases of hemolytic streptococcal disease, and in 1 patient with typhoid fever. Unfortunately, this patient was not fol- 


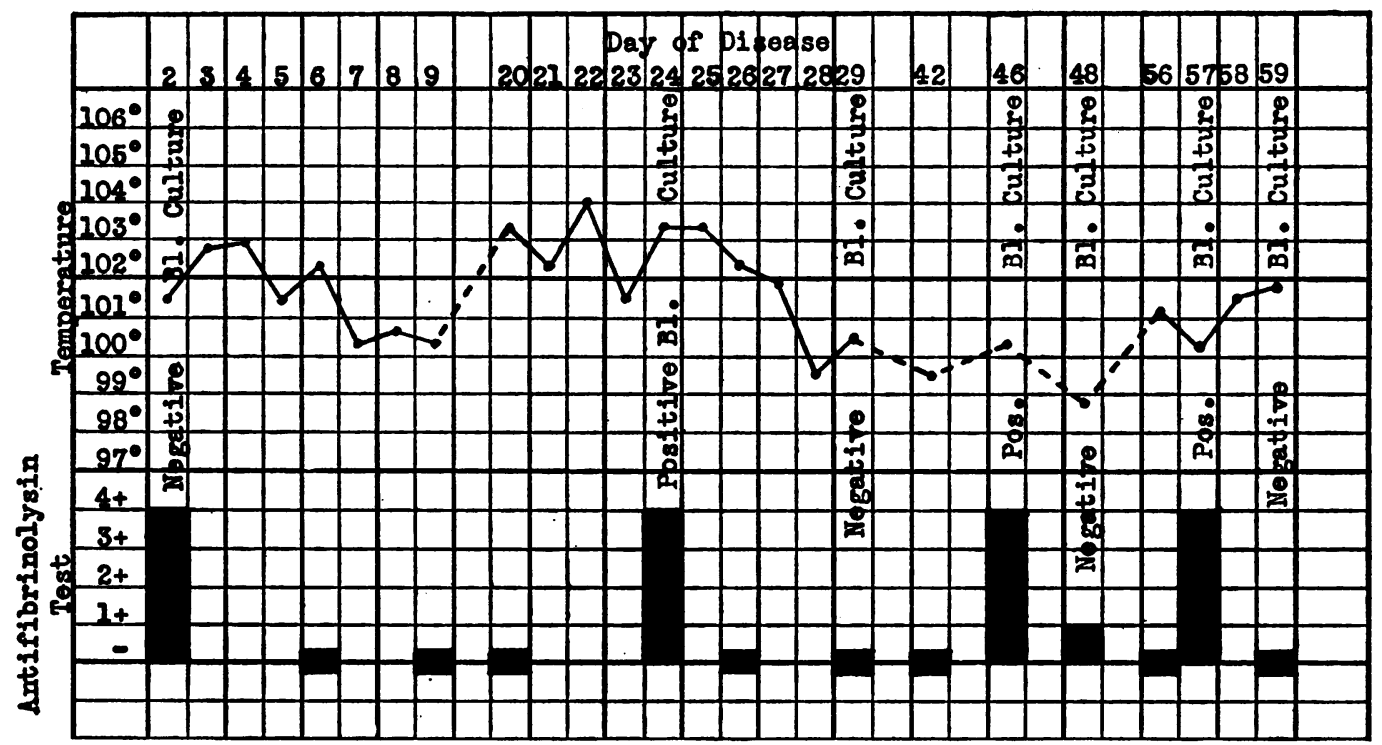

Chart 6. Patient R. S. Age 11 Years. Diagnosis: Congenital Heart Disease with Subacute Bacterial Endocarditis (Streptococcus Viridans)

Child, known to have congenital heart disease, was admitted on the second day of his acute illness with cough, bloody sputum, chills and high fever. Signs on physical and roentgenological examination were those of congenital heart disease with pulmonary infarction. There were no petechiae seen and admission blood culture was negative. Repeated nose and throat cultures showed no hemolytic streptococci. On the twenty-fourth day of illness streptococcus viridans was cultured from the blood stream. Child continued to have bouts of fever associated with petechiae and positive blood cultures throughout his seven months' period of hospitalization and course was gradually downhill. Postmortem examination verified the clinical diagnosis.

Antifibrinolysin tests. The test was strongly positive on admission and became negative in a few days. With each shower of bacteria into the blood stream, the test rapidly became positive and returned to negative when blood cultures showed no growth.
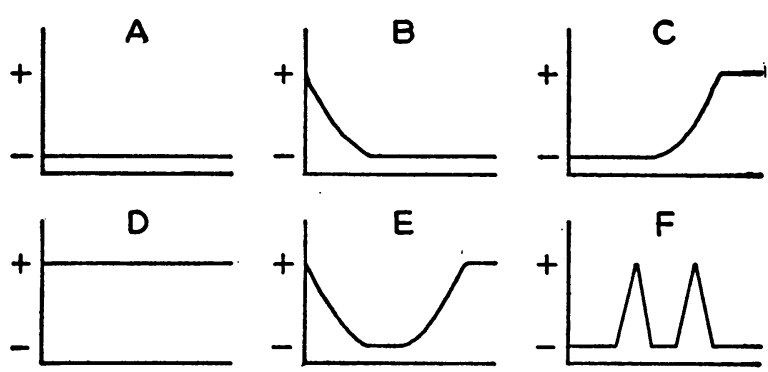

Chart 7. Trends of Antifibrinolysin Tests

Trend $A$, persistently negative tests; Trend $B$, positive to negative tests; Trend $C$, negative to positive tests; Trend $D$, persistently positive tests; Trend $E$, positive to negative to positive tests; Trend $F$, negative to positive to negative tests (spiking).

lowed through convalescence; it is possible that if the period of study had been longer the trend would have been $B$ rather than $D$.

Trend $\mathrm{E}$ (positive to negative to positive) ap- peared in 3 cases of non-streptococcal infection followed during convalescence by a streptococcal illness, and in the 2 cases in which the bacteriological findings suggested a possible simultaneous pneumococcal and hemolytic streptococcal infection.

Trend F (spiking) was encountered in only 1 instance: a case of congenital heart disease with streptococcus viridans endocarditis.

\section{DISCUSSION}

A study of the trend of the streptococcal antifibrinolysin test under various conditions of health and disease in individuals of the pediatric age-group demonstrates several interesting points.

The test does not seem to be influenced by minor variations in the child, which may occur either in health or in the presence of a mild infection. Tests were repeatedly negative in the 


\begin{tabular}{|c|c|c|c|c|c|c|}
\hline \multirow[b]{2}{*}{ Conditions studied } & \multicolumn{6}{|c|}{ Trends of antifibrinolysin test } \\
\hline & 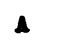 & B & c & $D$ & E & \\
\hline Normal.............................. & 15 & & & & & \\
\hline 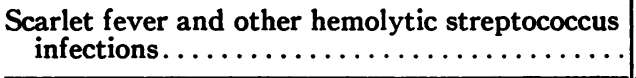 & 8 & 4 & 53 & 5 & (5) & \\
\hline Rheumatic fever and chorea $\ldots \ldots \ldots \ldots \ldots \ldots$ & 3 & & & 30 & & \\
\hline Acute nephritis........................... & 1 & & & 10 & & \\
\hline 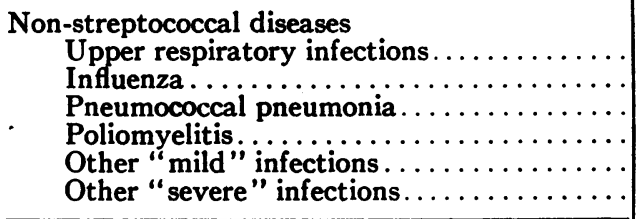 & $\begin{array}{r}6 \\
2 \\
4 \\
4 \\
20 \\
3\end{array}$ & $\begin{array}{r}3 \\
28 \\
3 \\
9\end{array}$ & & 1 & & 1 \\
\hline Newborns.. & & 6 & & & & \\
\hline
\end{tabular}

Chart 8. Trends of Antifibrinolysin Tests and Conditions Under Which They Were Observed

Note: Figures represent the number of cases; figure in parenthesis refers to cases of secondary, or possible seondary, hemolytic streptococcal infection.

normal infants and children reported, and single tests on a much larger series of healthy young individuals and of children with various types of non-infectious diseases have corroborated this finding. Consistently negative tests were also found in pediatric patients with mild non-streptococcal infections.

Newborn infants, however, furnished an exception to this rule. Strongly positive tests were obtained with venous blood of all 6 newborns and with their respective placental blood, in spite of the fact that tests on the mothers' venous blood, just prior to and just after delivery, were negative. In none of the mothers was there any indication of a recent hemolytic streptococcal infection. In 3 of the babies, the test had become negative by the tenth day of life, while in the remaining 3 , tests were still positive on the tenth day but were negative at 6 weeks of age. Lippard and Wheeler (11) obtained maximally positive tests in 14 of the 28 newborns whom they studied. It would be of much interest to ascertain if this is a true streptococcal antifibrinolysin which has been stored up in the fetus.

Positive tests were obtained in 67 of the 75 patients with hemolytic streptococcal infections at some time during the course of their illness and convalescence. In 53 of the 70 patients with primary hemolytic streptococcal infections, the test was negative during the acute phase of the disease and became positive about the time of recovery. This trend, Trend $\mathrm{C}$, (negative to positive), which was present in over 70 per cent of the cases studied, was seen in no other type of infection. Trend $E$ (positive to negative to positive), which was observed in 3 patients with secondary and 2 patients with probable secondary hemolytic streptococcal infections, appears to represent "hemolytic streptococcal " Trend C (negative to positive) preceded by "non-specific" Trend $B$ (positive to negative), such as might be expected in a hemolytic streptococcal infection complicating a non-streptococcal illness. Of the 8 patients in whom repeated tests were negative, 2 were infants, 2 were children who had a prolonged illness with complications, and 1 was an infant with a fatal infection. Tillett (3) found that in patients with prolonged or fatal streptococcal infections the test was apt to remain negative or to become only weakly positive, and Lippard and Johnson (12) report that the antibody response in infancy as determined by the 
antifibrinolysin and antistreptolysin content of the blood may be poorly developed.

Concerning the non-specific aspect of the antifibrinolysin test, there would seem to be little doubt that the more severe types of non-streptococcal infection are also capable of producing a positive test in children. Positive tests are common during the acute febrile stage of such diseases. It is of much interest, however, that the trend of the results was strikingly different from that seen in the majority of patients with hemolytic streptococcal infections. The mechanism for this production of positive tests in non-streptococcal infections is not apparent at this time. It might find an analogy in the increase in the streptococcidal power of the blood of patients in the active phase of infections due to a variety of organisms, as described by Tillett (13). It should be noted that Friedemann and Sutliff (14) found an unusual polysaccharide in the blood in similar conditions.

The presence of positive tests in rheumatic fever, chorea, and nephritis is in accord with the findings reported in the literature $(3,4,5,6,12$, $15,16,17,18)$. Six of our patients with rheumatic fever were retested 3 to 5 months after discharge from the hospital and all continued to show strongly positive tests although they had apparently been free from any significant infections. The positive tests seen during convalescence from uncomplicated hemolytic streptococcal infections such as scarlet fever were found to persist for several days or at the most a few weeks. This strong association of positive tests with rheumatic fever suggests that the antifibrinolysin test may be of diagnostic aid. Unfortunately, the presence or absence of an etiological relationship between the hemolytic streptococcus and rheumatic fever cannot be determined from the trend of the antifibrinolysin tests. The trend of the test in rheumatic fever is neither of the "non-specific" type, Trend B (positive to negative), nor of the " hemolytic streptococcal" type, Trend C (negative to positive). There are three points, however, which are consistent with such a relationship: (1) If rheumatic fever is a sequel to infection by the hemolytic streptococcus it would be anticipated that only the latter portion -the positive phase - of Trend $\mathrm{C}$ would be obtained; (2) the incidence of persistently positive tests (10 out of 11 patients) in this disease is remarkably similar to that observed in the cases of acute nephritis, all of which followed scarlet fever or other types of hemolytic streptococcal infection; (3) with the possible exception of one patient with typhoid fever, who was not followed after discharge from the hospital, in no disease other than rheumatic fever did the duration of positive tests at all approach that seen in nephritis.

\section{SUMMARY AND CONCLUSIONS}

The trends of repeated streptococcal antifibrinolysin tests in 15 normal infants and children, 6 newborns, and 203 patients of the pediatric agegroup are reported.

The study suggests that, although a single test may be of no diagnostic value in the pediatric laboratory unless viewed in the light of the history and bacteriological findings, the trend of several tests may be significant. The type of trend that was observed in the majority of patients with hemolytic streptococcal infections was found in no other type of disease.

\section{BIBLIOGRAPHY}

1. Tillett, W. S., and Garner, R. L., The fibrinolytic activity of hemolytic streptococci. J. Exper. Med., 1933, 58, 485.

2. Tillett, W. S., Edwards, L. B., and Garner, R. L., Fibrinolytic activity of hemolytic streptococci. The development of resistance to fibrinolysis following acute hemolytic streptococcus infections. J. Clin. Invest., 1934, 13, 47.

3. Tillett, W. S., The occurrence of antifibrinolytic properties in the blood of patients with acute hemolytic streptococcus infections. J. Clin. Invest., 1935, 14, 276.

4. Myers, W. K., Keefer, C. S., and Holmes, W. F., Jr., The resistance to fibrinolytic activity of the hemolytic streptococcus with special reference to patients with rheumatic fever and rheumatoid (atrophic) arthritis. J. Clin. Invest., 1935, 14, 119.

5. Waaler, Erik, Undershelser over de hemolytiske streptokokkers fibrinolytiske evne, og over tidstedevaerelsen av antifibrinolysiner i blod. Norsk mag. f. laegevidensk, 1936, 97, 449.

6. Waaler, Erik, Development of antifibrinolytic properties in blood of patients with rheumatic fever, chronic infective arthritis and bacterial endocarditis. J. Clin. Invest., 1937, 16, 145.

7. Furcolow, L., and Fousek, D., Streptococcal antifibrinolysin test in clinical use. J. Bact., 1936, 31, 102. 
8. Lancefield, R. C., A serological differentiation of human and other groups of hemolytic streptococci. J. Exper. Med., 1933, 57, 571.

9. Boisvert, P. L., Human hemolytic streptococci from diseases in childhood. Am. J. Dis. Child. (In press).

10. Estrada, Emilio, The grouping of hemolytic streptococci from children by means of the precipitin test of Lancefield. Yale J. Biol. and Med. (In press).

11. Lippard, V. W., and Wheeler, G. W., Beta hemolytic streptococcic infection in infancy and in childhood. III. Placental transmission of antifibrinolysin and antistreptolysin. Am. J. Dis. Child., 1936, 52, 61.

12. Lippard, V. W., and Johnson, P., Beta hemolytic streptococcic infection in infancy and in childhood. I. Antifibrinolysin and antistreptolysin response. Am. J. Dis. Child., 1935, 49, 1411.

13. Tillett, W. S., The bactericidal action of human serum on hemolytic streptococci. I. Observations made with serum from patients with acute infections and from normal individuals. II. Factors which influence the phenomenon in vitro. J. Exper. Med., 1937, 65, 147.

14. Friedemann, T. E., and Sutliff, W. D., Appearance of fermentable polysaccharide in the blood and a simple method for its detection. Science, 1939, 90, 335.

15. Hadfield, G., Magee, V., and Perry, C. B., The lysis of fibrin by streptococci: its application to the problems of rheumatic infection in children. Lancet, 1934, 1, 834.

16. Stuart-Harris, C. H., A study of hemolytic streptococcal fibrinolysis in chronic arthritis, rheumatic fever, and scarlet fever. Lancet, 1935, 2, 1456.

17. McEwen, C., Bunim, J. J., and Alexander, R. C., Bacteriologic and immunologic studies in arthritis. II. Results of various immunologic tests in different forms of arthritis. J. Lab. and Clin. Med., 1936, 21, 465.

18. Perry, C. B., The relationship between acute rheumatism and streptococcal antifibrinolysin. Arch. Dis. Childhood, 1939, 14, 32. 